Brauer, Hartmut; Ziolkowski, Marek; Haueisen, Jens:

Biomagnetische Stromdichterekonstruktion mit Hilfe von Minimum-NormVerfahren

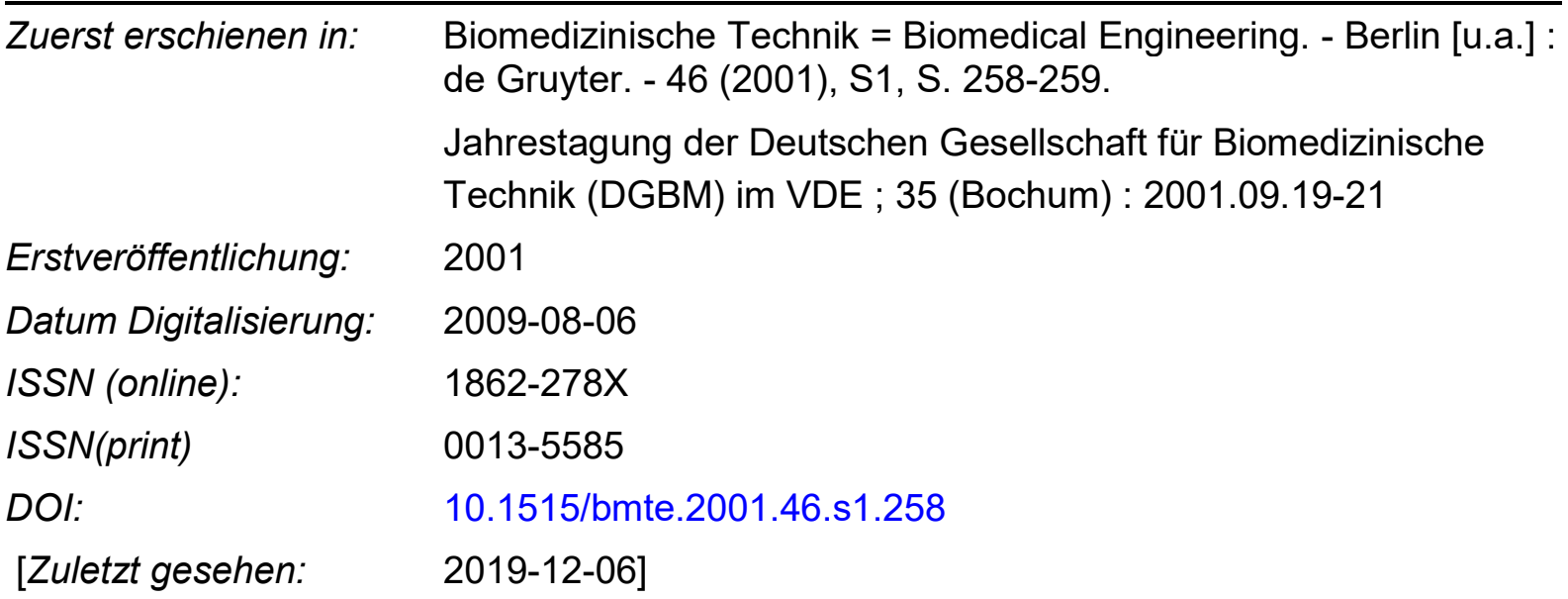

„Im Rahmen der hochschulweiten Open-Access-Strategie für die Zweitveröffentlichung identifiziert durch die Universitätsbibliothek IImenau."

"Within the academic Open Access Strategy identified for deposition by Ilmenau University Library."

„Dieser Beitrag ist mit Zustimmung des Rechteinhabers aufgrund einer (DFGgeförderten) Allianz- bzw. Nationallizenz frei zugänglich."

"This publication is with permission of the rights owner freely accessible due to an Alliance licence and a national licence (funded by the DFG, German

DFG

Research Foundation) respectively." 


\title{
BIOMAGNETISCHE STROMDICHTEREKONSTRUKTION MIT HILFE VON MINIMUM-NORM-VERFAHREN
}

\author{
H. Brauer', M. Ziolkowski ${ }^{2}$, J. Haucisen ${ }^{3}$ \\ 'Institut f. Allgemeine u. Theoretische Elektrotechnik, TU IImenau, Deutschland \\ ${ }^{2}$ Lehrstuhl f. Theoretische Elcktrotechnik u. Informatik, TU Stettin, Polen \\ ${ }^{3}$ Biomagnetisches Zentrum, Univ. Jena, Deutschland \\ brauer@e-technik.tu-ilmenau.de
}

\section{EINLEITUNG}

Stromdichterekonstruktionen der elektrischen Herzaktivität mit Hilfe der nichtinvasiv gewonnenen Magnetokardiogramme (MKG) stellen ein relativ neues und vielversprechendes Hilfsmittel für die kardiologische Diagnostik dar [1]. Ein typisches Resultat derartiger Rekonstruktionen sind meist farbkodierte Feldverteilungen, die den Amplituden der Stromdichte in einem Volumen bzw. auf einer Oberfläche entsprechen. Zur Identifikation der Stromquellenverteilungen in einem Volumenleiter, die die $\mathrm{zu}$ bewertenden magnetischen und/oder elektrischen Felder generiert haben, werden zahlreiche inverse Lösungsstrategien, vor allem Minimum-Norm-Verfahren, verwendet.

Die meisten dieser Methoden sind ursprünglich für die Lokalisation von Stromdipolen in dem elektrisch aktiven Gewebe entwickelt worden. Es ist jedoch bekannt, daß es sich bei diesen Feldquellen meist um ausgedehnte, also räumlich verteilte Quellen handelt und folglich Methoden zur Stromdichterekonstruktion angewendet werden müssen.

In früheren Arbeiten haben wir die Leistungsfähigkeit verschiedener inverser Algorithmen bei Verwendung eines 3D-Lösungsraumes untersucht [2,3]. Wir haben festgestellt, daß keiner dieser Algorithmen das inverse Problem zufriedenstellend lösen kann. Im diesem Beitrag soll daher die Leistungsfähigkeit von Minimum-Norm-Least-Square-Methoden, die auf einzelne Flächen als Lösungsraum für die Rekonstruktion angewendet werden, untersucht werden.

\section{MATERIALIEN UND METHODEN}

Wir haben mit Hilfe des Biomagnetometer-Systems (62 Kanäle) des Biomagnetischen Zentrums Jena Magnetfeldverteilungen an einem homogenen TorsoPhantom, in das cin Modell einer ausgedehnten Stromquelle eingebracht wurde, gemessen. In die flächenhafte, rechteckige Stromquelle (Abmessungen $50 \mathrm{~mm} x$ $60 \mathrm{~mm}$, Abb. 1) wurde ein Strom von $500 \mu \mathrm{A}$ und einer Frequenz von $25 \mathrm{~Hz}$ cingeprägt. Zusätzlich wurden von 67 Elektroden Oberflächenmappings aufgezeichnet.
Als Lösungsraum für die Stromdichterekonstruktion wurden einzelne Ebenen gewählt, die ein regelmäßiges Gitter von $21 \times 21$ Knoten bilden. Der Abstand von zwei Nachbarknoten wie auch der Abstand zwischen den Rekonstruktionsebenen beträgt $10 \mathrm{~mm}$ (Abb. 1).
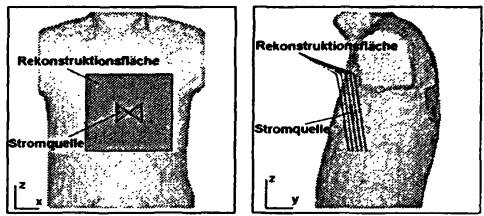

Abb. 1: Torso-Phantom mit ausgedehnter Stromquelle und fünf parallelen Rekonstruktionsebenen.

In jedem Knoten wird ein Stromdipol fixiert, die Dipolmomente und ihre Orientierungen werden dann mit Minimum-Norm-Least-Square-Methoden bestimmt. Die verwendeten Lösungsverfahren sind im Detail bereits in $[2,3]$ beschrieben worden.

Wir haben Stromdichteverteilungen anhand von magnetischen, elektrischen bzw. kombinierten Meßdaten rekonstruiert. Dabei bestand der Lösungsraum für die Rekonstruktion jedoch nur aus einer Ebene, die Schritt für Schritt um jeweils $10 \mathrm{~mm}$ verschoben wurde (siehe Abb. 1, rechts). Die Ebenen wurden so gewählt, $\mathrm{da} \beta$ die tatsächliche Position der Quellenanordnung mit der der mittleren Ebene übereinstimmte.

Um die rekonstruierten Stromdichteverteilungen miteinander vergleichen zu können, haben wir eine Visualisierungstechnik angewendet, die auf der Berechnung von äquivalenten Ellipsoiden beruht [4]. Dabei werden aus der Menge aller rekonstruierten Stromdipole diejenigen ausgewählt, deren Momente einen vorgegebenen Schwellwert überschreiten. Daraus wird dann ein äquivalenter Ellipsoid ermittelt, der diese Stromdipole optimal umschließt. Seine Halbachsen ermöglichen eine Abschätzung der Ausdehnung der Quellenverteilung im Raum. Diese Ellipsoide stellen damit eine mögliche Beschreibungsform für verteilte (ausgedehnte) Quellenstrukturen dar. 


\section{ERGEBNISSE}

Die Untersuchungen haben gezeigt, daß sowohl Ort als auch Ausdehnung des physikalischen Quellenmodells auf Einzelebenen besser rekonstruiert werden als im früher betrachteten 3D-Lösungsraum [2,3].
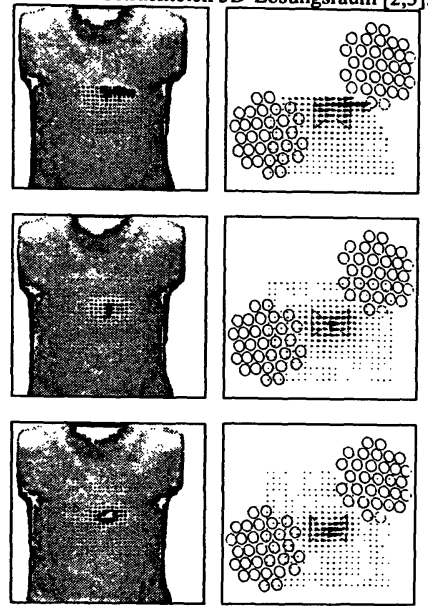

Abb. 2: Rekonstruktion einer ausgedehnten Stromquelle im homogenen Torso-Phantom; Frontalansicht (links) für die oberflächennahe Ebene (oben), die wahre Quellenebene (mitte) bzw. die tiefliegende Ebene (unten) mit den magnetischen Sensorpositionen (rechts)

In den Abbildungen 2 und 3 sind die Resultate der besten Rekonstruktionen wiedergegeben, die mit Hilfe von kombinierter (magnetisch + elektrisch) Meßdaten gewonnen wurden.

Tabelle 1 zeigt die Resultate der Stromdichterekonstruktion bei Verwendung von elektrischen Daten (E), magnetischen Daten (M) sowie kombinierten Daten $(M+E)$ für fünf verschiedene Ebenenpositionen.

Tab. 1: Abstănde zwischen dem COG und dem Zentrum der Quelle für verschiedene Rekonstruktionsebenen

\begin{tabular}{c|ccccc}
\hline Meß- & \multicolumn{5}{|c}{ Rekonstruktionsebene } \\
daten & $-20 \mathrm{~mm}$ & $-10 \mathrm{~mm}$ & $0 \mathrm{~mm}$ & $10 \mathrm{~mm}$ & $20 \mathrm{~mm}$ \\
\hline $\mathrm{E}$ & 21.3 & 20.0 & 7.0 & 11.1 & 20.1 \\
$\mathrm{M}$ & 23.0 & 11.8 & 24.1 & 29.5 & 28.7 \\
$\mathrm{M}+\mathrm{E}$ & 25.4 & 10.4 & 5.2 & 10.8 & 20.4 \\
\hline
\end{tabular}

Die schlechten Rekonstruktionsergebnisse für dic magnetischen Daten sind dadurch bedingt, $\mathrm{daB}$ die Sensorflächen des magnetischen Meßsystems nur einen kJeinen Teil der relevanten Feldinformation erfassen. Der geringste Abstand ergab sich, wenn mit kombinierten Daten direkt in der Rbene rekonstruiert wurde, in der sich dic Originalquelle befand. In diesem
Fall sind die größten Halbachsen des äquivalenten Ellipsoids gleich $81.8 \mathrm{~mm}$ und $49.8 \mathrm{~mm}$, was gut mit den Abmessungen des das Quellenmodell umfassenden Rechteckes ( $60 \mathrm{~mm}$ breit, $50 \mathrm{~mm}$ hoch) übereinstimmt.
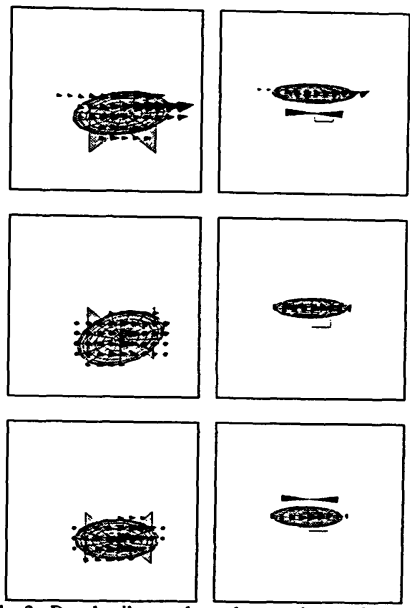

Abb. 3: Beschreibung der rekonstruierten Stromdichteverteilung durch äquivalente Ellipsoide; (Positionen entsprechen denen in Abb. 2)

\section{SCHLUSSFOLGERUNGEN}

Wir haben festgestellt, daß Minimum-Norm-LeastSquare-Methoden für die Lősung inverser Probleme geeignet sind, wenn die Rekonstruktionsebene nicht weit entfernt von der Position der wahren Stromquelle gewählt wird und sowohl elektrische als auch magnetische Daten verwendet werden. Darüber hinaus sind die äquivalenten Ellipsoide geeignet, Rekonstruktionsresultate zu visualisieren. Damit ist es besser möglich, die räumliche Ausdehnung der Quellenanordnungen zu beschreiben.

\section{LITERATURHINWEISE}

[1] U. Leder, J. Haueisen, M. Huck, H. Nowah, „Noninvasive imaging of arrhythmogenic left-ventricular myocardium after infarction", The Lancet, vol. 23, p 234, 2000

[2] H. Brauer, M. Ziolkowski, J. Haucisen, „Verification of extended sources reconstruction techniques using a torso phantom", COMTEL, vol. 20, No. 2, pp. 595-606, 2001

[3] H. Braucr, M. Ziolkowski, J. Haucisen, " Evaluation of inverse field solutions with biomedical applications", COMPEL, vol. 20, No. 3, pp. $665-675,2001$

[4] M. Ziolhowski, J. Haucisen, H. Nouah, H. Brauer, "Equivalent Fillipsoid as an interpretation tool of evtended current distributions in biomagnetic inverse problems". IIEEI? Trans. on Magnetics, vol. 36, pp. 1692-45, 2000) 